\title{
VERIFICAÇÃO DA EFICÁCIA DE DIFERENTES INSETICIDAS NO CONTROLE AMBIENTAL DE TUNGA PENETRANS (L., 1758)
}

\author{
Ricardo Soares Matias
}

\begin{abstract}
Referências sobre o controle de Tunga penetrans a nivel ambiental têm sido feitas, sem contudo se estabelecer critérios para avaliar a eficácia dos produtos. Neste trabalho usamos as variaçōes das prevalências obtidas antes e após o controle químico, como instrumento de avaliação. Foram testados os piretróides cipermetrina na formulação de concentrado emulsionável, $C E$, (Cymperator 25CE) a 0,2\% e 0,5\%; deltametrina na formulaçâo de concentrado emulsionável $C E$, (K-Othrine 25CE) a 0,6\% e deltametrina na formulação de concentrado de suspensão ou flowable concentrate, $S C,(K$-Othrine $50 S C)$ a $0,3 \%$. O resultado deste trabalho nâo se baseia apenas na performance dos produtos testados, verificada através da variação de prevalência, mas também em outros aspectos relevantes em saúde pública como a relação custo/beneficio; subsídios fundamentais em campanhas de saúde pública.
\end{abstract}

Palavras-chaves: Tungiase. Controle. Resistência. Tunga penetrans.

Os bolsões gerados nas periferias das cidades, eram inicialmente constituídos de imigrantes rurais atraidos pela magia destas, enxergando nelas um futuro promissor. Junto veio toda uma cultura particular que com o tempo vai se expressando na ruralização do meio urbano, que aliada à falta de equipamentos e serviços urbanos favorecem o desenvolvimento de inúmeros microhabitats onde se desenvolvem vetores, roedores e zoonoses.

Hoje, estes bolsões passam, também, a receber imigrantes do próprio meio urbano, resultado de uma situação econômica que os empurra para a periferia das cidades na tentativa de acomodar sua situação econômica com a necessidade de sobreviver.

As enfermidades transmitidas por vetores têm sido uma das cargas mais pesadas suportadas pela humanidade, que pela sua endemicidade ou epidemicidade, tem afetado seriamente a vida do homem nas regiões tropicais 9 .

A tungiase, zoonose que tem como agente Tunga penetrans, tem se apresentado como importante endemia em nosso estado, ampliando junto com outras doenças transmissiveis nosso quadro morboso.

Os vetores e agentes causadores de zoonoses têm se apresentado como inimigos contra os quais, muitas vezes, a luta tem sido inglória. Esta dificuldade

Divisão de Zoonoses e Vetores da Secretaria da Saúde e do Meio Ambiente do Rio Grande do Sul, Porto Alegre, RS. Endereço para correspondência: Dr. Ricardo Soares Matias. Av. Angélica 2121/131, Santa Cecilia, 01227 São Paulo, SP.

Recebido para publicação em 24/08/90. se dá, por exemplo, em função da capacidade adaptativa do inseto frente aos inseticidas.

Estes têm se mostrado tão adaptáveis às mudanças ambientais que são os únicos organismos que disputam com o homem o dominio do planeta. Esta capacidade de adaptação foi eloqüentemente descrita por Holland (1905) citado por Martin Dress GG e Nájera-Morrondo JA (1973): "Quando a lua tiver desaparecido do firmamento e as calotas polares tiverem avançado até o Equador, sobre um fragmento de líquem que cresça nas rochas próximas às neves perpétuas do Panamá, se encontrará um inseto movendo suas antenas na débil luz do gasto sol, que representará a única manifestação de vida na terra"9.

Apesar dos clorados terem se apresentado como importantes controladores de pragas, seu uso tem sido proscrito tanto pelos efeitos danosos ao ambiente como à saúde, como também pelo fato de induzirem com maior intensidade à resistência em função de sua maior persistência no ambiente, mantendo a pressão seletiva por semanas ou meses 11 .

Assim, a indústria tem desenvolvido cada vez mais a formulação de novos grupos de inseticidas como os piretróides sintéticos e outros como os reguladores de crescimento, bactérias e vírus.

Hoje em dia sabemos que, em saúde pública, não podemos prescindir do uso de inseticidas para o controle destes organismos. Entretanto pesquisas devem ser feitas no sentido de se conhecer bem seu efeito controlador nos diferentes agentes.

No caso da tungíase devemos considerar dois momentos para seu controle; um é o controle ambiental, assunto deste trabalho, procurando, através de inseticidas eliminar o agente do solo. Aliado a isto 
devemos procurar meios alternativos de controle numa forma integrada como saneamento, controle de reservatórios não humanos e educação para a saúde. $O$ outro é o controle no indivíduo parasitado.

No primeiro caso os cuidados são de impacto ambiental e indução à resistência. Quanto à resistência o cuidado deve ser grande uma vez que sendo uma zoonose de conhecimento recente mas de alta morbidade, tem levado a um controle químico intenso $e$ persistente.

No segundo caso, apesar da enucleação ser o método conhecidamente mais eficaz, referências têm sido feitas para o controle em reservatórios humanos e não humanos, de produtos como o DDT a $5 \% \mathrm{em}$ benzeno e DDT a $5 \%$ em vaselina 8 ; gamotox a $0,004 \%(\mathrm{BHC})^{3}$; óleo de caju13; pedra do inferno, bálsamo do Peru ou limão ${ }^{6}$; repelentes ${ }^{1}$; BHC e DDT nas meias como preventivo 12 .

As referências existentes sobre o controle deste parasita não fornecem resultados, apenas suposições. Assim é o caso de Gordon (1941) ${ }^{4}$ e Jolly $(1926)^{7}$ que consideram que aposentos e terrenos arejados e limpos reduzem o risco de infestação.

Gordon (1941) ainda orienta na utilização da solução de Waterson para o controle ambiental que consiste numa emulsão de sabão, água e óleo ${ }^{4}$.

Bruce $(1942)^{2}$ recomenda para o controle do parasita no solo antissépticos, enquanto que Harman $(1972)^{5}$ sugere a aplicação de DDT; Bel e col. $(1979)^{1}$ orienta na utilização de organofosforados e Jolly $(1926)^{7}$ sugere o uso de solução de querosene.

Disto fica clara a necessidade de se investigar diferentes produtos quimicos no controle deste parasita, verificando concentrações e doses capazes de controlar a densidade parasitária em niveis epidemiologicamente aceitáveis.

\section{MATERIAL E MÉTODOS}

O trabalho se desenvolveu em 18 núcleos residenciais urbanos e rurais de dois municípios do Rio Grande do Sul (Torres, a $210 \mathrm{Km}$ de Porto Alegre pela BR 101 e Capão da Canoa a $140 \mathrm{Km}$ da capital pela mesma rodovia, ambos no litoral norte do estado). Não houve uma razão especial para a escolha dos locais, pois eram localidades que estavam dentro de nossa programação de trabalho para o controle da tungíase, tendo sido aproveitadas para a realização do ensaio. Foi realizado um diagnóstico da situação através de investigação epidemiológica casa a casa em toda a área, em agosto, outubro e dezembro de 1988, momento em que também foi realizado o controle químico. A avaliação de resultados também foi feita através de investigação epidemiológica casa a casa em maio, junho e agosto de 1989.
A investigação epidemiológica foi feita usando a metodologia tradicional através de fichas especificas, com uma parte verificando dados pessoais como endereço, de onde vieram, se havia tungiase, como tratam o local onde vivem, se há animais infectados, etc., para se conhecer a dinâmica da zoonose e uma outra parte levantando nome, idade de cada um dos familiares e quando se infectaram, além da região do corpo afetada, para se conhecer a prevalência e sazonalidade da infeç̧ão.

A avaliação é feita usando apenas a segunda parte. Com isto temos a prevalência antes e depois do controle químico.

Os inseticidas foram usados nas seguintes doses: Cymperator $25 \mathrm{CE}$ nas doses de $2 \mathrm{ml} / 1$ e $5 \mathrm{ml} / 1$; KOthrine 25CE na dose de $6 \mathrm{ml} / 1$ e K-Othrine 50SC na dose de $3 \mathrm{ml} / 1$. A recomendação das doses foi feita pelos fabricantes e a escolha destes produtos foi em função de serem produtos que a nossa Divisão já utilizava para o controle de outros insetos.

O controle químico foi feito pulverizando-se a área externa de todas as residências das diferentes áreas, ruas, terrenos baldios, residências abandonadas ou em construção (internã e externamente). Uma segunda aplicação foi feita ao redor de vinte dias após o fim da primeira aplicação. Este tempo é em função do ciclo do parasita, pois a ação inseticida se faz basicamente sobre o adulto. Na Vila do Faxinal em Torres, o intervalo entre as aplicações foi de sete dias em função do tempo disponivel para realizarmos o trabalho.

Neste ensaio não realizamos outras atividades paralelas como enucleação de reservatórios humanos, controle de cães, treinamento de professores e alunos, pelo fato de que não poderíamos, pela falta de recursos, desenvolver todas estas atividades em todos os locais, o que causaria maiores dificuldades na interpretação dos dados.

Para esta fase de controle usamos um pulverizador tipo carrinho, marca Hatsuta G150 com motor Branco CB035, 3.5 HP com pulverizador Hatsuta S-25, capacidade 25 litros/minuto e pressão de 0-700 $\mathrm{lb} / \mathrm{pol}^{2}$, movido a gasolina, com pistola HG e bico cone G2, utilizando mangueira para alta pressão hidráulica com bitola $3 / 8^{\prime \prime}$, calibrada para uso em 200 libras de pressão, o que nos dá um rendimento de 150 litros de solução para cada $3936 \mathrm{~m}^{2}$, aproximadamente.

Após um período que variou de sete a doze meses após o segundo controle químico, fizemos uma avaliação através de nova investigação epidemiológica a fim de verificarmos a prevalência e percentual de infestação e traçarmos, a partir destes dados, as variações ocorridas. Tivemos o cuidado de que o periodo de verão estivesse entre a fase de controle e avaliação, pois é neste periodo que ocorre um elevado número de casos. 
Uma das etapas do trabalho era a de verificar a relação custo/beneficio de uma campanha desta natureza, para verificarmos, ao final da campanha, o que significaria o custo por pessoa protegida. Para isto tomamos uma localidade onde realizamos o controle com determinado produto. A partir dai avaliamos, teoricamente, o gasto com outros inseticidas e diferentes concentrações de uso para um mesmo inseticida, para traçarmos a relação comparativa para cada um deles.

Para a realizaçāo destes cálculos fizemos, como um dia de trabalho, cinco horas consecutivas de atividades. Durante este periodo realizamos os seguintes gastos discriminados abaixo:

\begin{tabular}{lc}
\hline Volume de solução gasto/dia & $1050 \mathrm{lt}$ \\
Área tratada/dia & $27552 \mathrm{~m}^{2}$ \\
Combustivel gasto nos pulverizadores/dia & $17,5 \mathrm{lt}$ \\
Óleo de dois tempos usado nos pulverizadores/dia & $1 \mathrm{lt}$ \\
Combustível usado no veiculo para transporte/dia & $8,3 \mathrm{lt}$ \\
Número total de residências pulverizadas & 3886 \\
População estimada na área sob controle & 18303 \\
\hline
\end{tabular}

Para o cálculo total das despesas realizadas, devemos considerar dois momentos. O primeiro é o do controle propriamente dito, que leva, neste caso em particular, dez dias. Como fizemos duas aplicações, levamos vinte dias nesta atividade, onde tivemos despesas com diárias pagas a um motorista, um técnico e cinco auxiliares de campo, além de gasolina para o veiculo de transporte de pessoal e dos pulverizadores, óleo de dois tempos para as máquinas pulverizadoras e o inseticida gasto.

Para o segundo momento, diagnóstico e avaliação, levamos cinco dias para cada uma destas etapas, havendo despesas quanto às diárias para o mesmo grupo acima descrito e combustivel apenas para o veiculo.

\section{RESULTADOS}

A Tabela 1 relaciona todos os gastos efetuados na campanha, beneficiando uma população estimada em 18.303 pessoas. A partir dai traçamos a relação custo/benefício para cada produto testado, verificando o custo por pessoa, cujos resultados são fundamentais na eleição do produto a ser usado em futuras campanhas, comparando sua eficiência com o custo.

Tabela 1 -Discriminação dos gastos éfetuados, visando estabelecer a relacão custo/beneficio, segundo diferentes inseticidas testados.

\begin{tabular}{lccccc}
\hline & \multicolumn{4}{c}{ Inseticidas } \\
\cline { 2 - 6 } Itens analisados & $\mathrm{A}$ & $\mathrm{B}$ & $\mathrm{C}$ & $\mathrm{D}$ & $\mathrm{E}$ \\
\hline Dose & $6 \mathrm{ml} / 1$ & $3 \mathrm{ml} / 1$ & $2 \mathrm{ml} / 1$ & $5 \mathrm{ml} / 1$ & $10 \mathrm{ml} / 1$ \\
Concentração & $25 \mathrm{~g} / 1$ & $50 \mathrm{~g} / 1$ & $250 \mathrm{~g} / 1$ & $250 \mathrm{~g} / 1$ & $240 \mathrm{~g} / 1$ \\
Vol. inseticida/máquina & $900 \mathrm{ml}$ & $450 \mathrm{ml}$ & $300 \mathrm{ml}$ & $750 \mathrm{ml}$ & $1500 \mathrm{ml}$ \\
Área tratada/máquina & $3936 \mathrm{~m}^{2}$ & $3936 \mathrm{~m}^{2}$ & $3936 \mathrm{~m}^{2}$ & $3936 \mathrm{~m}^{2}$ & $3936 \mathrm{~m}^{2}$ \\
mg i.a./m & 05,72 & 05,72 & 19,05 & 47,64 & 91,46 \\
Vol. inseticidade/dia & 06,031 & 03,151 & $02,01 \mathrm{1}$ & 05,251 & 10,051 \\
Vol. inseticida/20 dias & 1261 & 631 & 421 & 1051 & 2101 \\
Custo/litro de inset\$ & 43,61 & 86,75 & 65,06 & 65,06 & 15,05 \\
Custo de inset./20 dias & 5495,42 & 5465,06 & 2732,53 & 6831,33 & 3160,12 \\
Custo combustivel/30 dias & 325,76 & 325,76 & 325,76 & 325,76 & 325,76 \\
Diárias pagas/30 dias & 4145,35 & 4145,35 & 4145,35 & 4145,35 & 4145,35 \\
Custo total/30 dias $\$$ & 9966,53 & 9936,17 & 7203,64 & 11302,44 & 7631,23 \\
População beneficiada & 18303 & 18303 & 18303 & 18303 & 18303 \\
Relação custo/beneficio & 0,54 & 0,54 & 0,39 & 0,59 & 0,42 \\
\hline
\end{tabular}

SValores em dólares

$\mathrm{A}=\mathrm{K}$-Othrine $25 \mathrm{CE} . \mathrm{B}=\mathrm{K}$-Othrine $50 \mathrm{SC} . \mathrm{C}=$ Cymperator $25 \mathrm{CE}$ na dose de $2 \mathrm{ml} / 1$

$\mathrm{D}=$ Cymperator $25 \mathrm{CE}$ na dose de $5 \mathrm{ml} / 1 . \mathrm{E}=$ Dursban $2 \mathrm{E}$ (este produto não foi discutido no trabalho em funçāo de ter sido aplicado em apenas uma localidade faltando informações para um estudo mais detalhado, entretanto foi aqui incluido para se ter uma idéia comparativa dos custos com um fosforado). 
Matias RS. Verificação da eficácia de diferentes inseticidas no controle ambiental de Tunga penetrans $(L ., 1758)$. Revista da Sociedade Brasileira de Medicina Tropical24: 31-36, jan-mar, 1991

A Tabela 2 nos mostra as variações de percentuais de prevalência e infestação obtidos antes e depois do controle para cada um dos produtos testados e as variações médias para cada grupo de localidades. Os valores negativos, obtidos, indicam a redução na infecção e infestação.

Tabela 2 - Distribuição das localidades testadas, segundo as variações de infestação e infecção por inseticida usada, no Rio Grande do $S u l$.

\begin{tabular}{|c|c|c|c|c|c|c|}
\hline \multirow{2}{*}{ Localidades } & \multirow{2}{*}{ Data } & \multicolumn{2}{|c|}{ Residências } & \multicolumn{2}{|c|}{ Populaçāo } & \multirow{2}{*}{ Produto/dose } \\
\hline & & $\%$ infest & $\%$ & $\%$ infec & $\%$ & \\
\hline $\begin{array}{l}\text { S. Pedro }{ }^{1}(A C)^{3} \\
\text { S. Pedro }(A p C)^{4}\end{array}$ & $\begin{array}{l}12 / 88 \\
08 / 89\end{array}$ & $\begin{array}{l}58,33 \\
17,65\end{array}$ & $-69,74$ & $\begin{array}{l}43,01 \\
12,99\end{array}$ & $-69,80$ & Cymperator $25 \mathrm{CE} 2 \mathrm{ml} / 1^{5}$ \\
\hline $\begin{array}{l}\text { Rio Dentro }^{1}(\mathrm{AC}) \\
\text { Rio Dentro }(\mathrm{ApC})\end{array}$ & $\begin{array}{l}12 / 88 \\
08 / 89\end{array}$ & $\begin{array}{r}100,00 \\
46,67\end{array}$ & $-53,23$ & $\begin{array}{l}94,95 \\
48,05\end{array}$ & $-49,39$ & Cymperator $25 \mathrm{CE} 2 \mathrm{ml} / 1$ \\
\hline $\begin{array}{l}\text { S. Rita }(A C) \\
\text { S. Rita }(A p C)\end{array}$ & $\begin{array}{l}12 / 88 \\
08 / 89\end{array}$ & $\begin{array}{l}77,78 \\
15,38\end{array}$ & $-80,23$ & $\begin{array}{l}62,96 \\
12,90\end{array}$ & $-79,51$ & Cymperator $25 \mathrm{CE} 2 \mathrm{ml} / 1$ \\
\hline $\begin{array}{l}\text { S. João² (AC) } \\
\text { S. João (ApC) }\end{array}$ & $\begin{array}{l}12 / 88 \\
08 / 89\end{array}$ & $\begin{array}{l}85,71 \\
75,00\end{array}$ & $-12,50$ & $\begin{array}{l}89,29 \\
65,00\end{array}$ & $-27,20$ & Cymperator 25CE $2 \mathrm{ml} / 1$ \\
\hline $\begin{array}{l}\text { Total (AC) } \\
\text { Total (ApC) }\end{array}$ & & $\begin{array}{l}77,61 \\
30,61\end{array}$ & $-60,56$ & $\begin{array}{l}69,77 \\
28,81\end{array}$ & $-58,71$ & Cymperator 25CE $2 \mathrm{ml} / 1$ \\
\hline $\begin{array}{l}\text { Caiu do céu }{ }^{2}(\mathrm{AC}) \\
\text { Caiu do céu }(\mathrm{ApC})\end{array}$ & $\begin{array}{l}10 / 88 \\
05 / 89\end{array}$ & $\begin{array}{l}45,31 \\
19,48\end{array}$ & $-57,01$ & $\begin{array}{l}34,57 \\
12,31\end{array}$ & $-64,51$ & Cymperator $25 \mathrm{CE} 5 \mathrm{ml} / 1$ \\
\hline $\begin{array}{l}\text { S. Luzia II }(\mathrm{AC}) \\
\text { S. Luzia II (ApC) }\end{array}$ & $\begin{array}{l}10 / 88 \\
06 / 89\end{array}$ & $\begin{array}{l}57,78 \\
33,96\end{array}$ & $-41,23$ & $\begin{array}{l}42,21 \\
22,80\end{array}$ & $-45,98$ & Cymperator $25 \mathrm{CE} 5 \mathrm{ml} / 1$ \\
\hline $\begin{array}{l}\text { Faxinal }^{2}(\mathrm{AC}) \\
\text { Faxinal (ApC) }\end{array}$ & $\begin{array}{l}10 / 88 \\
08 / 89\end{array}$ & $\begin{array}{l}87,50 \\
69,09\end{array}$ & $-21,04$ & $\begin{array}{l}76,77 \\
63,46\end{array}$ & $-17,34$ & Cymperator $25 \mathrm{CE} 5 \mathrm{ml} / 1$ \\
\hline $\begin{array}{l}\text { Salinas }^{2}(\mathrm{AC}) \\
\text { Salinas (ApC) }\end{array}$ & $\begin{array}{l}08 / 88 \\
08 / 89\end{array}$ & $\begin{array}{l}34,04 \\
20,69\end{array}$ & $-39,22$ & $\begin{array}{l}30,77 \\
20,00\end{array}$ & $-35,00$ & Cymperator $25 \mathrm{CE} 5 \mathrm{ml} / 1$ \\
\hline $\begin{array}{l}\text { Total (AC) } \\
\text { Total (ApC) }\end{array}$ & & $\begin{array}{l}53,92 \\
32,24\end{array}$ & $-40,21$ & $\begin{array}{l}41,62 \\
23,50\end{array}$ & $-43,54$ & Cymperator $25 \mathrm{CE} 5 \mathrm{ml} / 1$ \\
\hline $\begin{array}{l}\text { Zona Nova }{ }^{2}(\mathrm{AC}) \\
\text { Zona Nova }(\mathrm{ApC})\end{array}$ & $\begin{array}{l}10 / 88 \\
06 / 89\end{array}$ & $\begin{array}{l}73,63 \\
29,76\end{array}$ & $-59,58$ & $\begin{array}{l}54,19 \\
24,31\end{array}$ & $-55,14$ & K-Othrine $25 \mathrm{CE} 6 \mathrm{ml} / 1^{5}$ \\
\hline $\begin{array}{l}\text { S. Luzia } I^{2}(A C) \\
\text { S. Luzia I (ApC) }\end{array}$ & $\begin{array}{l}10 / 88 \\
05 / 89\end{array}$ & $\begin{array}{l}29,55 \\
23,64\end{array}$ & $-20,00$ & $\begin{array}{l}20,63 \\
17,70\end{array}$ & $-14,20$ & K-Othrine $25 \mathrm{CE} 6 \mathrm{ml} / 1$ \\
\hline $\begin{array}{l}\text { P. } \mathrm{Cal}^{2}(\mathrm{AC}) \\
\text { P. Cal }(\mathrm{ApC})\end{array}$ & $\begin{array}{l}08 / 88 \\
08 / 89\end{array}$ & $\begin{array}{l}77,19 \\
50,00\end{array}$ & $-35,22$ & $\begin{array}{l}72,31 \\
35,90\end{array}$ & $-50,35$ & K-Othrine $25 \mathrm{CE} 6 \mathrm{ml} / 1$ \\
\hline $\begin{array}{l}\text { G. Vargas } I^{2}(A C) \\
\text { G. Vargas II }(A p C)\end{array}$ & $\begin{array}{l}08 / 88 \\
08 / 89\end{array}$ & $\begin{array}{l}26,86 \\
10,00\end{array}$ & $-62,77$ & $\begin{array}{l}14,92 \\
05,74\end{array}$ & $-61,53$ & K-Othrine $25 \mathrm{CE} 6 \mathrm{ml} / 1$ \\
\hline $\begin{array}{l}\text { Total (AC) } \\
\text { Total (ApC) }\end{array}$ & & $\begin{array}{l}48,72 \\
25,43\end{array}$ & $-49,86$ & $\begin{array}{l}36,30 \\
18,18\end{array}$ & $-49,92$ & K-Othrine $25 \mathrm{CE} 6 \mathrm{ml} / 1$ \\
\hline $\begin{array}{l}\text { S. Jorge } 2(\mathrm{AC}) \\
\text { S. Jorge }(\mathrm{ApC})\end{array}$ & $\begin{array}{l}10 / 88 \\
06 / 89\end{array}$ & $\begin{array}{l}44,09 \\
06,32\end{array}$ & $-85,67$ & $\begin{array}{l}28,13 \\
02,40\end{array}$ & $-91,47$ & K-Othrine $50 \mathrm{SC} 3 \mathrm{ml} / 1$ \\
\hline $\begin{array}{l}\text { Curtume }^{2}(\mathrm{AC}) \\
\text { Curtume (ApC) }\end{array}$ & $\begin{array}{l}08 / 88 \\
08 / 89\end{array}$ & $\begin{array}{l}57,26 \\
37,09\end{array}$ & $-35,23$ & $\begin{array}{l}50,10 \\
25,20\end{array}$ & $-49,70$ & K-Othrine $50 \mathrm{SC} 3 \mathrm{ml} / 1$ \\
\hline $\begin{array}{l}\mathrm{Stan}^{2}(\mathrm{AC}) \\
\mathrm{Stan}(\mathrm{ApC})\end{array}$ & $\begin{array}{l}08 / 88 \\
08 / 89\end{array}$ & $\begin{array}{l}69,62 \\
13,24\end{array}$ & $-80,98$ & $\begin{array}{l}22,50 \\
09,93\end{array}$ & $-55,87$ & K-Othrine $50 S C 3 \mathrm{ml} / 1$ \\
\hline $\begin{array}{l}\text { Joāo XXIII }^{1} \text { (AC) } \\
\text { João XXIII (ApC) }\end{array}$ & $\begin{array}{l}08 / 88 \\
08 / 89\end{array}$ & $\begin{array}{l}87,50 \\
62,96\end{array}$ & $-28,05$ & $\begin{array}{l}82,84 \\
45,45\end{array}$ & $-45,14$ & K-Othrine $50 S C 3 \mathrm{ml} / 1$ \\
\hline $\begin{array}{l}\text { G. Vargas } I^{2}(A C) \\
\text { G. Vargas I }(A p C)\end{array}$ & $\begin{array}{l}08 / 88 \\
08 / 89\end{array}$ & $\begin{array}{l}43,75 \\
22,81\end{array}$ & $-47,86$ & $\begin{array}{l}37,37 \\
13,95\end{array}$ & $-62,67$ & K-Othrine $50 S C 3 \mathrm{ml} / 1$ \\
\hline $\begin{array}{l}\text { Total (AC) } \\
\text { Total (ApC) }\end{array}$ & & $\begin{array}{l}55,04 \\
25,05\end{array}$ & $-54,49$ & $\begin{array}{l}39,85 \\
16,59\end{array}$ & $-58,37$ & K-Othrine $50 S C 3 \mathrm{ml} / 1$ \\
\hline
\end{tabular}

1 = Zona rural, 2 = Zona urbana, $3=$ Percentuais obtidos antes do controle químico (AC), $4=$ Percentuais obtidos após o controle químico (ApC), $5=$ Piretróides. 


\section{DISCUSSÃO}

Apesar de a literatura referir-se praticamente ao uso de organoclorados, seja em hospedeiros como no ambiente, fica descartada a continuação de seu uso, tanto pelos riscos à saúde e ao ambiente, como também por exercerem uma pressão muito intensa na seleção de resistentes 351215 .

Convém citar aqui o fato de que a limpeza e arejamento preconizados por Gordon (1941) 4 e Jolly $(1926)^{7}$, não constitui, de forma alguma, controle de Tunga penetrans, uma vez que encontramos este parasita em locais de bom nivel sócio-econômicocultural com uma preocupação muito grande na limpeza das residências e do peridomicilio como na Praia da Cal e na Getúlio Vargas I e II.

Os inseticidas quimicos têm sido um importante componente no controle integrado nos programas contra os vetores. $O$ desenvolvimento de resistência aos inseticidas é a maior ameaça tanto aos especialistas no controle de vetores como à indústria química. Portanto, há uma constante necessidade de se procurar e testar, observando o custo-efetividade, novos inseticidas quimicos para fornecer alternativas para o controle de vetores $^{8}$. Além disto a OMS (1976) 10 sugere a investigação de novos pares de compostos a serem usados alternadamente ou em mistura com piretróides sintéticos, posição compartilhada por Priester \& Georghiou (1978) ${ }^{14}$.

O que se observa na Tabela 2 é que praticamente não há diferença nas variaçōes médias dos percentuais obtidos para os grupos de inseticidas Cymperator 25CE a $0,5 \%$; K-Othrine $25 \mathrm{CE}$ a $0,6 \%$ e o K-Othrine $50 \mathrm{SC}$ a $0,3 \%$. O mesmo não corre entre estes e o Cymperator $25 \mathrm{CE}$ a $0,2 \%$

É importante observar que devem interferir muitas variáveis, tanto a nível de susceptiveis como de ambiente na variação da prevalência, pois, por exemplo, se observarmos a Vila Zona Nova e a Santa Luzia I, apesar de serem favelas típicas, com características muito semelhantes, apresentam uma redução de prevalência bem diferentes, tendo sido usado o mesmo produto e a mesma concentração.

Dentro deste mesmo raciocínio está a diferença obtida com o Cymperator 25CE, onde obtivemos uma redução média da prevalència maior a $0,2 \%$ do que a $0,5 \%$. Talvez uma explicação para este fato seja a de que a concentração a $0,2 \%$ tenha sido usada na zona rural e a $0,5 \%$ na zona urbana com maior possibilidade de reinfestação. Caso seja verdadeiro, isto também explicaria seu melhor desempenho em relação aos outros produtos.

Entretanto esta observação fica prejudicada na medida em que a localidade João XXIII (zona rural) onde usamos K-Othrine 50SC a 0,3\%, obtivemos um resultado muito ruim na redução dos índices de prevalência, bem pior que as outras localidades rurais onde foi usado o Cymperator $25 \mathrm{CE}$ a $0,2 \%$ (São Pedro, Rio de Dentro e Santa Rita). Isto tudo mostra a necessidade de se continuar testes desta natureza.

Podemos dizer que estes produtos testados são eficazes no controle de Tunga penetrans, uma vez que não há história de controle químico deste agente de forma sistemática, não permitindo, ainda, a seleção de resistentes. Mas mesmo assim, a performance obtida com os diferentes produtos foi perceptivel. Entretanto em campanhas de saúde pública, deve-se aliar a este aspecto o custo da campanha, observando o custo do inseticida por diluição e por metro quadrado. Assim sendo, estas observações mostram que o Cymperator $25 \mathrm{CE}$ na dose de $0,2 \%$ se mostrou mais efetivo no controle deste agente.

\section{SUMMARY}

In this study the variation of infection prevalence before and after control procedures was utilized as an evaluation tool. The pyrethroids Cypermethrine (Cymperator 25EC), deltamethrine ( $\mathrm{K}$-Othrine 25EC and 5OSC) were tested. Apart from the effects, the results evaluation has also been based on the cost per area and cost benefit, which are relevant items in Public Health campaigns. We believe all these aspects will help us to evaluate these results and serve as basis for future better insecticide choices. Resistence.

Key-words: Tungiasis. Tunga penetrans. Control.

\section{REFERÊNCIAS BIBLIOGRÁFICAS}

1. Bell A, Neely CL, Peeples J. Tungiasis in Tennessee. Southern Medical Journal 72: 141-143, 1979.

2. Bruce GO, Knigin TD, Yolles SF. A Discussion of chigoe (Tunga penetrans) based on experiences in British Guiana. The Military Surgeon 91: 446-452, 1942.

3. Cooper JE. An outbreak of Tunga penetrans in a pig herd. Veterinary Record 80: 365-366, 1967.

4. Gordon RM. Jigger flea. The Lancet 2: 47-49, 1941.

5. Harman RRM. Tungiasis. In: Raak A (ed) Textbook of dermatology, 2nd edition, Blackell, London p. 855, 1972.

6. Henning G. Zur geschichte des sandflohs (Sarcopsylla penetrans L) in Afrika. Naturwissenschftliche wochenschrift 19: 310-312, 1904.

7. Jolly GC. An entomological episode of the east african campaign. The Indian Medical Gazette 61: 164-165, 1926.

8. Lim JL, Visvalingam M. Relative potency of lambdacyalothrin and cypermethrin applied as thermal fogs for the control houseflies (Musca domestica) and mosquitoes (Aedes aegypti). ICI Agriculture Reserch Centre. Lock Bag № 1006, GPO 75990 Malacca, Malaysia, 1989. 
Matias RS. Verificação da eficácia de diferentes inseticidas no controle ambiental de Tunga penetrans (L., 1758). Revista da Sociedade Brasileira de Medicina Tropical 24: 31-36, jan-mar, 1991

9. Martin DGG, Nájera-Morrondo JA. Agricultura, Malaria y el uso de plaguicidas. Boletin de la Oficina Sanitaria Panamericana 74: 469-478, 1973.

10. Organización Mundial de la Salud. Resistencia de vectores y reservorios de enfermedades a los plaguicidas. Série de Informes Técnicos No 585, 1976.

11. Organización Mundial de la Salud. Resistencia de los vectores a los plaguicidas. Boletín de la Oficina Sanitaria Panamericana 86: 442-451, 1979.

12. Pessoa SB, Viana A. Parasitologia médica. 9a edição, Guanabara Koogan, Rio de Janeiro p. 814-815, 1976.
13. Piso G. História natural e médica das Índias Ocidentais. Ministério da Educação e Cultura, Rio de Janeiro, Livro 4 p. $278,1957$.

14. Priester TM, Georghiou GP. Induction of high resistence to permethrin in Culex pipiens quinquefasciatus. Journal of Economic Entomology 71: 197-200, 1978.

15. Vaz Z, Rocha UF. Tunga penetrans (L. 1758). "Bicho do pé" em gado bovino. Importância clínica, observações patológicas e tratamento pelo DDT. Livro de homenagem a R.F. Almeida 40: 327-332, 1946. 